\title{
Highly Tunable Molecularly Doped Flexible Poly(dimethylsiloxane) Foam Piezoelectric Energy Harvesters
}

\section{Christopher Petroff, Thomas Bina, Geoffrey Hutchison}

Submitted date: 02/08/2019 - Posted date: 02/08/2019

Licence: CC BY-NC-ND 4.0

Citation information: Petroff, Christopher; Bina, Thomas; Hutchison, Geoffrey (2019): Highly Tunable Molecularly Doped Flexible Poly(dimethylsiloxane) Foam Piezoelectric Energy Harvesters. ChemRxiv. Preprint.

Despite considerable research interest in developing piezoelectric materials, little work has focused on the fundamental design of these materials from the ground up. Herein, we present a general, versatile method for producing tunable, flexible piezoelectric energy harvesters (PEHs) with excellent piezoelectric response. Using a poly(dimethylsiloxane) (PDMS) foam derived from a sugar template, we separate the electrical and mechanical properties of the PEH, thereby allowing us to optimize them separately. The electrical properties were tuned by varying the poling field, the polar dopant, and the dopant concentration. The mechanical properties were tuned by varying foam preparation and thus the compressive modulus. Through the careful tuning of these properties, we are able to achieve a maximum piezoelectric response of 153 $\mathrm{pC} / \mathrm{N}$ - considerably higher than most other reported flexible PEHs. Combined with our previous work, we demonstrate that doping polymer foams with polar dopants is a highly general strategy and has the potential to lead to materials with considerably higher piezoelectric responses.

File list (4)

pdms-foam-paper.pdf (1.28 MiB)

view on ChemRxiv - download file

si.pdf (281.20 KiB) view on ChemRxiv - download file tex-files.zip (0.92 MiB) view on ChemRxiv - download file peak-finding-script.py (9.23 KiB) view on ChemRxiv - download file 


\title{
Highly Tunable Molecularly Doped Flexible Poly(dimethylsiloxane) Foam Piezoelectric \\ Energy Harvesters
}

\author{
Christopher A. Petroff, Thomas F. Bina, and Geoffrey R. Hutchison* \\ Department of Chemistry, University of Pittsburgh, 219 Parkman Avenue, Pittsburgh, \\ Pennsylvania 15260, United States \\ E-mail: geoffh@pitt.edu
}

\begin{abstract}
Despite considerable research interest in developing piezoelectric materials, little work has focused on the fundamental design of these materials from the ground up. Herein, we present a general, versatile method for producing tunable, flexible piezoelectric energy harvesters (PEHs) with excellent piezoelectric response. Using a poly(dimethylsiloxane) (PDMS) foam derived from a sugar template, we separate the electrical and mechanical properties of the PEH, thereby allowing us to optimize them separately. The electrical properties were tuned by varying the poling field, the polar dopant, and the dopant concentration. The mechanical properties were tuned by varying foam preparation and thus the compressive modulus. Through the careful tuning of these properties, we are able to achieve a maximum piezoelectric response of $153 \mathrm{pC} / \mathrm{N}$ - considerably higher than most other reported flexible PEHs. Combined with our previous work, we demonstrate that doping polymer foams with polar dopants is a highly general strategy and has the potential to lead to materials with considerably higher piezoelectric responses.
\end{abstract}




\section{Keywords}

piezoelectric, flexible, PDMS, energy harvester, tunable

\section{Introduction}

Piezoelectric energy harvesters (PEHs) have the potential to self-power a growing class of small, low-power electronic devices such as remote or wearable sensors or Internet of Things devices. ${ }^{1}$ Based on the direct piezoelectric effect, these devices have the ability to generate electricity scavenged from the environment or the human body to power devices indefinitely and to eliminate the environmental and labor costs of battery replacements. ${ }^{2,3}$ The vast majority of piezoelectric materials are hard ceramics (Young's modulus, $\left.E_{Y}>100 \mathrm{GPa}\right)^{4,5}$ such as lead zirconium titanate (PZT). ${ }^{6,7}$ As such, these hard, brittle materials are only able to withstand a very limited range of deformations, limiting their application in many situations. ${ }^{6,8,9}$ Most current polymer piezoelectric materials, such as poly(vinylidene fluoride) (PVDF) ( $d_{33}$ of $\left.20-30 \mathrm{pC} / \mathrm{N}\right)$, have a piezoelectric response an order of magnitude or more below that of PZT $(>200 \mathrm{pC} / \mathrm{N}) \cdot{ }^{6}$ Consequently, there is a need for intrinsically flexible materials with high electromechanical response.

A variety of approaches towards new flexible piezoelectric materials have been developed, including

peptide nanotubes,${ }^{10}$ sponge-like ceramic-polymer composites, ${ }^{11}$ quantum dot-polymer composites, ${ }^{12}$ nanoparticle-polymer composites, ${ }^{13}$ and sugar-nanofiber composites. ${ }^{14}$ Despite long being an area of considerable research interest, few attempts have been made at the rational design of these materials from the ground up to maximize their piezoelectric response and efficiency. A key figure of merit is the $d$ piezoelectric tensor, as well as the electromechanical coupling factor, $k$, which is a quantitative measure of the efficiency of piezoelectric materials at interconverting electrical and mechanical energy. ${ }^{15}$ The general coupling efficiency of a piezoelectric material is ${ }^{16,17}$

$$
k=d \sqrt{\frac{E_{Y}}{\epsilon}}
$$

where $k$ is the coupling efficiency, $d$ is the piezoelectric coefficient, $E_{Y}$ is the Young's modulus, and $\epsilon$ 
is the relative permittivity. Since perfect efficiency cannot be reached, it follows that: ${ }^{16}$

$$
d<\sqrt{\frac{\epsilon}{E_{Y}}}
$$

As the relative permittivity is directly related to the dipole moment, ${ }^{18}$ this relation shows that, if the material polarization is generated separately from the matrix, each property can be separately tuned for desired parameters. The bulk dipole moment can be tuned by incorporating different polar dopants and by the degree of polar alignment. The Young's modulus is a measure of the stiffness of a material along the axis of deformation and can be tuned by changing the properties of the matrix. It is known that lowering the modulus of a material increases its piezoelectric response, but, by separating the two factors, this can be more fully exploited. ${ }^{16,19}$

In this study, building on our previously published work, ${ }^{16}$ we present a highly tunable, flexible $\mathrm{PEH}$ that demonstrates the effect of both the bulk dipole moment and the modulus on piezoelectric response. While the maximum piezoelectric response we report here is somewhat lower than our previous work, we have vastly improved the consistency and tailorability of our system, while simultaneously showing that our method is broadly applicable and not derived from matrix specific effects. By doping poly(dimethylsiloxane) (PDMS) foam with varying amounts of highly polar small organic molecules and electrically poling at a range of voltages, we are able to control the bulk polarization of our PEH; similarly, by varying the curing temperature of our PDMS matrix, we are able to tune the modulus of our PEH. Our results demonstrate that each method can modulate the overall piezoelectric response as expected.

\section{Experimental Methods}

\section{Materials}

2-Chloro-4-nitroaniline (>98\%) (CNA) and 4-nitro-1,2-phenylenediamine (>97.0\%) were obtained from TCI. 2-Methyl-4-nitroaniline (99\%) (MNA) and 3-aminobenzoic acid (99+\%) were obtained from Acros Organics. N,N-Dimethyl-4-nitroaniline (>98\%), 4-nitroacetanilide (98\%), and acetanilide 
(98\%) were obtained from Alfa Aesar. Benzoic acid (99.5\%) was obtained from J.T. Baker. Acetonitrile ( $\geq 99.9 \%)$ and 1,4-dibromobenzene (98\%) were obtained from Sigma-Aldrich. Sodium chloride (certified ACS, crystalline) was obtained from Fisher Chemical. Sylgard 184 silicone elastomer system (polydimethylsiloxane; PDMS) was obtained from Dow Corning. Granulated sugar was obtained from Domino Foods. All chemicals were used as received. Ultrapure water $(18.2 \mathrm{M} \Omega \mathrm{cm})$ was generated using a Millipore Synergy system.

\section{PDMS Foam Preparation}

PDMS foams were produced from a sugar template using a procedure adapted from the literature. ${ }^{20}$ To prepare the sugar template, $5.2 \mathrm{~g}$ of white granulated sugar was mixed with $300 \mu \mathrm{L}$ of ultrapure water and pressed into a $30 \mathrm{~mm}$ diameter, $6 \mathrm{~mm}$ high mold using medium-firm pressure; excess sugar was scraped off the top to leave a level surface. After several hours, the sugar template was removed from the mold and allowed to continue drying for a total of at least $24 \mathrm{~h}$. PDMS was prepared according to the manufacturer's directions by mixing $10 \mathrm{~g}$ elastomer base with $1 \mathrm{~g}$ curing agent. After mixing, the PDMS mixture was added to a $9 \mathrm{~cm}$ diameter Petri dish containing four sugar templates. The Petri dish was placed under vacuum $(40 \mathrm{kPa})$ for a total of $5 \mathrm{~h}$. After $4 \mathrm{~h}$, the vacuum oven was switched on, and, over the course of the last hour, the samples were heated to $80{ }^{\circ} \mathrm{C}$. After heating, the samples were removed from the vacuum oven. The sugar was then removed by washing with ultrapure water under ultrasonication. Excess water was squeezed from the polymer foams, and the samples were placed in a $130{ }^{\circ} \mathrm{C}$ oven for $1 \mathrm{~h}$ to dry. They were then removed from the Petri dish, and a $20 \mathrm{~mm}$ diameter circular punch was taken from each sample. The average size of the sugar granules used to produce the template was approximately $0.3 \mathrm{~mm}$; therefore, we expect the template to produce open-cell foams with a similar pore size. Sugar granule size was measured from optical microscopy images taken on an Olympus BH2 microscope and analyzed with the Image software package (Version $1.48 \mathrm{k})$.

To vary the modulus of the foams, a similar procedure was followed by substituting sodium chloride templates, while varying the curing temperature. For the template, $5.3 \mathrm{~g}$ of sodium chloride was mixed with $250 \mu \mathrm{L}$ of ultrapure water. Freshly mixed PDMS was added, and the samples were 
placed in a vacuum desiccator for $4 \mathrm{~h}$. They were then removed from the desiccator and either left on the bench top at room temperature for $48 \mathrm{~h}$ or placed in a $100{ }^{\circ} \mathrm{C}$ or $200{ }^{\circ} \mathrm{C}$ oven for $1 \mathrm{~h}$; this produced three different sets - each with a different modulus. After curing and washing, excess water was squeezed from the foams, and they were placed in a vacuum desiccator to dry.

\section{Device Preparation and Electrical Poling}

In the second step of the process, dopant molecules were dissolved in acetonitrile. Foam samples were placed on the poling slides (described below), and $1 \mathrm{~mL}$ of the dopant solution was added to the foam sponge using a micropipet (e.g. $0.3 \mathrm{M}$ CNA samples had $1 \mathrm{~mL}$ of $0.3 \mathrm{M} \mathrm{CNA} /$ acetonitrile solution added to them). All solvent evaporated from the foams within $2 \mathrm{~h}$ leaving the dopant coating the pores of the PDMS foam (Figure S1). Samples were then electrically poled for $48 \mathrm{~h}$; shorter poling times led to inconsistent results whereas longer poling times (up to longest tested of $5 \mathrm{~d}$ ) were consistent with poling for $48 \mathrm{~h}$. All samples were $20 \mathrm{~mm}$ diameter, $6 \mathrm{~mm}$ thick circular disks.

For poling, samples were placed between two $5 \mathrm{~cm} \times 5 \mathrm{~cm}$ custom designed and manufactured printed circuit boards (PCBs) (Where Labs/DirtyPCBs.com) with $3.5 \mathrm{~cm} \times 3.5 \mathrm{~cm}$ electroless nickel immersion gold (ENIG) finished copper pads; polyimide tape was applied to the PCBs for protection. Samples were poled under a DC potential of $0 \mathrm{kV}-5 \mathrm{kV}$. During poling, the PCBs had a fixed spacing of $6 \mathrm{~mm}$. The poling slides were held in place using screws and wing-nuts, and separation was maintained through the use of aluminum spacers.

\section{Device Testing and Characterization}

After poling was completed, the samples were tested in a quasi-static manner, and the piezoelectric response was calculated. For testing, samples were placed between two PCBs identical to those used for poling; polyimide tape was not used as it was found to produce erroneous electrostatic signals. The sandwich was then positioned in a testing apparatus consisting of a threaded rod controlled by a stepper motor. A force sensor (Tekscan FlexiForce A201), with a PDMS spacer 
on top, rested above the sample sandwich and below the rod. A slight preload pressure $(\approx 0.3 \mathrm{~N})$ was applied using the threaded rod to minimize triboelectric charge generation; higher preload forces were seen to reduce the piezoelectric response. Using the apparatus, compressions of varying force (up to $10 \mathrm{~N}$ ) were applied along the Z-axis every few seconds; the rate of compression was held constant at approximately $1.7 \mathrm{~mm} / \mathrm{s}$. Force and current measurements were recorded for $70 \mathrm{~s}$ using either a Keithley 2612 SourceMeter or a Keithley 2614B SourceMeter; results were consistent between instruments. To ensure accuracy, the system was tested on commercial ceramic samples of PZT-4 (Steiner \& Martins SM121) and barium titanate (Steiner \& Martins SM511); the measured piezoelectric responses of $316 \pm 13 \mathrm{pC} / \mathrm{N}$ for PZT-4 and $134 \pm 5 \mathrm{pC} / \mathrm{N}$ for barium titanate agree favorably with the manufacturer's specifications of $300 \mathrm{pC} / \mathrm{N}$ and $140 \mathrm{pC} / \mathrm{N}$, respectively. A preload force of $10 \mathrm{~N}$ was used when testing the ceramics.

The collected data are simply the applied force and measured current - both as a function of time. To calculate the piezoelectric coefficient $\left(d_{33}\right)$, force versus charge was plotted for each compression, and the slope of the linear best fit was calculated. A custom Python script was used to compute charge by integrating the current peaks over time. The script identifies force peaks and subtracts off any baseline force; it then looks for the corresponding current peak and integrates it to calculate the corresponding charge during the period of increasing applied force.

Compression testing was performed using an Anton Paar MCR 302 rheometer. Applied force was increased from $0.2 \mathrm{~N}$ to $12.0 \mathrm{~N}$ over the course of $350 \mathrm{~s}$. As no linear region was observed for the sugar templated samples, the modulus was approximated by taking the secant of the stress-strain curve for strains corresponding to $0.2 \mathrm{~N}-10.0 \mathrm{~N}$ of applied force. ${ }^{21}$ For the sodium chloride templated samples, the slope of the linear region of the stress-strain curve was used. Masses were measured using a Denver Instrument SI-234 Summit Series analytical balance.

\section{Results and Discussion}

PDMS foams were produced from a sugar mold; doped with a solution of a polar, small organic molecule in acetonitrile; and electrically poled. This new system has several key advantages over 
our previously published polyurethane system: improved consistency and increased tailorability. ${ }^{16}$ The use of a sugar template allows for much more consistent foams to be produced; the foams have a more uniform pore size, density, and, thus, compressive modulus. As the polyurethane system was limited to dopants that could be dissolved in the polyol, the use of acetonitrile (or numerous other organic solvents) allows for a much wider array of dopants and dopant concentrations to be used. While we find a somewhat lower maximum piezoelectric response than in our previous report, this is likely due, in part, to the switch from flexible copper tape electrodes to smooth, rigid PCBs; wrinkles in the tape could amplify the response but lead to more inconsistent data. Additionally, in the present work, dopants are only able to coat the voids in the PDMS whereas they were more fully incorporated into the polyurethane. The PCBs, combined with smooth, consistent compressions driven by a stepper motor, have allowed us to greatly reduce error and produce smooth, tight charge-force curves from which to determine the piezoelectric response. Future applications could use flexible electrodes to obtain higher electrical response.

Figure 1A illustrates a schematic diagram of the testing procedure. As the foam is compressed by the stepper motor, the dipole concentration in the foam increases, causing charge to build up on either side and current to flow. The resulting force and current is measured simultaneously (Figure 1B); for each force peak, the corresponding current is integrated to compute the resultant charge. Note that the peak current remains constant with varying force since the rate of compression is intentionally held constant. Charge is plotted as a function of force (Figure 1C); the slope of the linear fit is the piezoelectric response, $d_{33}$. The results presented here are calculated from applied forces of up to $4 \mathrm{~N}$, as this was the most linear region of the charge-force curve; an analysis using applied forces of up to $10 \mathrm{~N}$ can be found in the Supporting Information. At higher forces (above $5 \mathrm{~N}-6 \mathrm{~N}$ ), the resultant charges increased sublinearly-likely due to the elastic properties of the foams. When higher forces are included in the analysis, the piezoelectric response is smaller, but the same trends are observed as discussed below (Figures S2 and S3). While other electrostatic effects may also be present, the linear/sublinear charge-force curve demonstrates that the measured response is primarily piezoelectric.

In Figure 2A, the piezoelectric response is plotted as a function of the concentration of the 

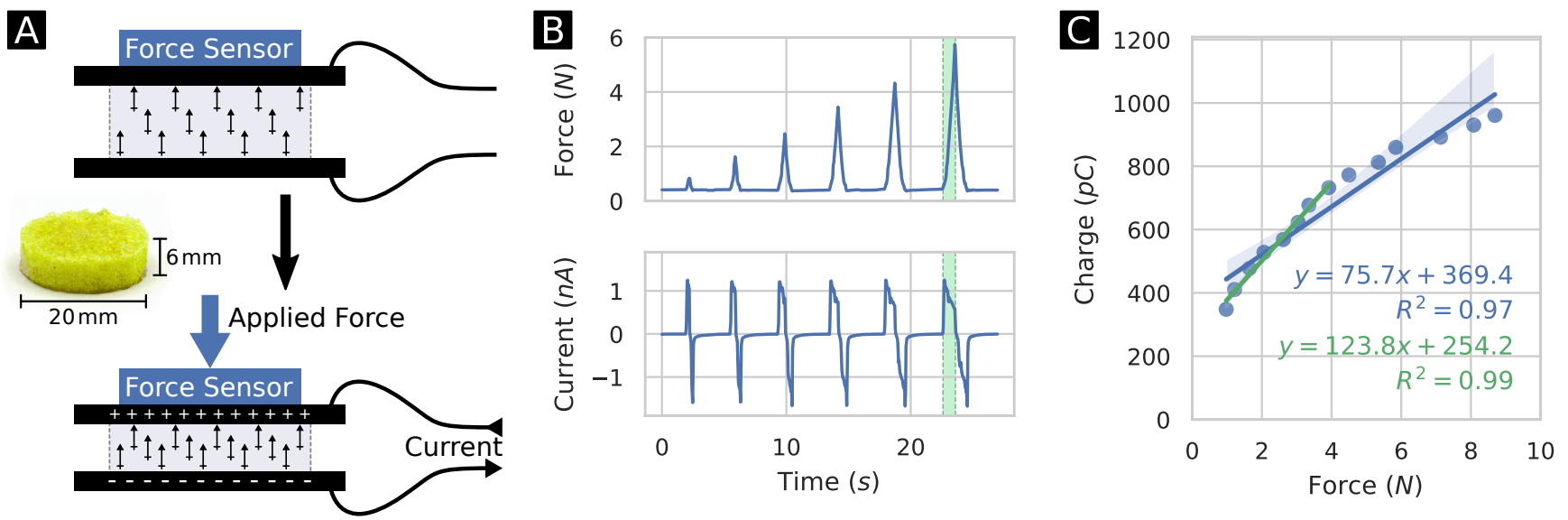

Figure 1: (A) Schematic diagram showing an applied force compressing the sample, increasing the dipole concentration, and leading to the buildup of charge and subsequent flow of current. Photograph of a representative foam with dimensions labeled (inset). (B) Simultaneous measurement of force and current over time. The green shading represents the time over which the current is integrated to calculate charge. (C) Charge-force plot of a $0.2 \mathrm{M}$ CNA doped sample poled at $800 \mathrm{~V} / \mathrm{mm}$. Primary analysis uses forces up to $4 \mathrm{~N}$ (green line).

added CNA dopant solution over a variety of poling fields while in Figure 2B, a subset of the data is presented showing the piezoelectric response as a function of the poling field. The response was tuned by varying the concentration of the added CNA dopant solution and by varying the poling voltage; it should be noted that the solvent evaporates early in the poling process leaving the dopant coating the foam's pores. For lower poling voltages $(\leq 400 \mathrm{~V} / \mathrm{mm})$, the piezoelectric response increased slightly (average piezoelectric response increase of $20 \%$ per $0.1 \mathrm{M}$ increase in dopant solution concentration) with increased dopant solution concentration. For higher poling voltages $(\geq 600 \mathrm{~V} / \mathrm{mm})$, the piezoelectric response peaked at a dopant solution concentration of $0.1 \mathrm{M}-0.2 \mathrm{M}$ before decreasing with higher concentrations; on average, the piezoelectric response of these samples increased by $34 \%$ per $200 \mathrm{~V} / \mathrm{mm}$ increase in poling field. A maximum piezoelectric response of $153 \mathrm{pC} / \mathrm{N}$ was observed for $0.1 \mathrm{M}$ CNA doped samples poled at $800 \mathrm{~V} / \mathrm{mm}$. The observed piezoelectric response increases with increased poling field as the molecular dipole moments become more aligned (Figure 2B). We were not able to test poling fields above $800 \mathrm{~V} / \mathrm{mm}$ due to power supply limitations, but the piezoelectric response will increase until dielectric breakdown is reached or full polar alignment is achieved. The dielectric strength of PDMS is $19 \mathrm{kV} / \mathrm{mm}$, but we expect the dielectric strength of our foams to be lower due to air present in the voids. ${ }^{22}$ The piezoelectric 
response does not always increase with increased dopant concentration, possibly due to interactions between the dopant and the PDMS matrix to which it adheres. PDMS is known to have an innate electrical response, although the mechanism behind it has been debated. ${ }^{23-25}$
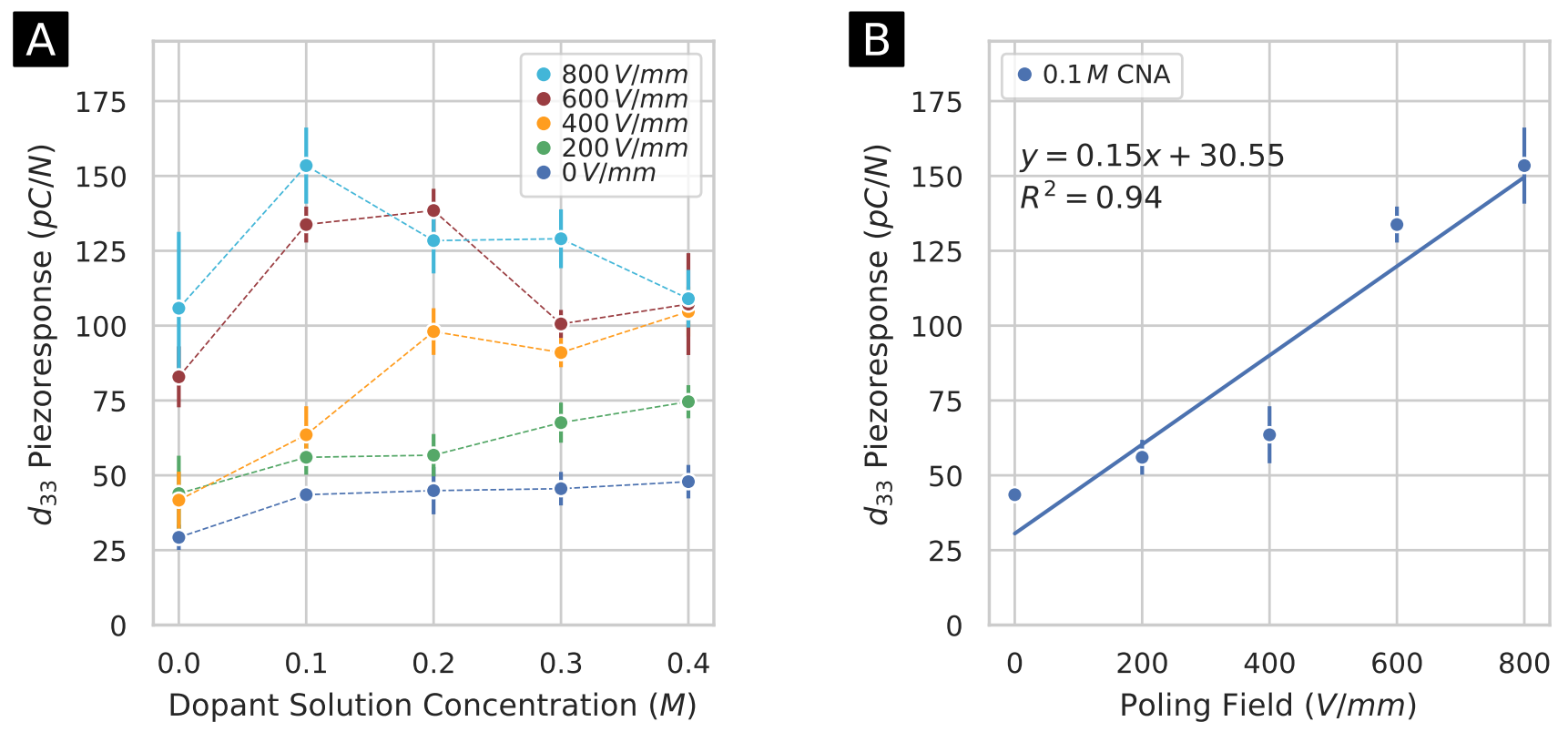

Figure 2: (A) The piezoelectric response as a function of added CNA dopant solution concentration over an array of poling fields. (B) The $0.1 \mathrm{M}$ CNA dopant solution subset showing the piezoelectric response increasing as a function of poling field. Error bars represent standard error across at least four samples.

As shown in Figure 3, the piezoelectric response varies as a function of dopant molecule dipole moment; the structures, names, and dipole moments of the dopants used are also given. The bulk piezoelectric response does not correlate directly with the dipole moment, but seems to initially increase before plateauing for dopants with dipoles above $4 \mathrm{D}$; on average, the piezoelectric response for dopants with dipole moments above $4 \mathrm{D}$ is $12 \%$ higher than those with smaller dipole moments and $75 \%$ higher than undoped foams. These data additionally demonstrate that the response cannot be attributed to the presence of piezoelectric crystals, since benzoic acid, 3-aminobenzoic acid, acetanilide, and 4'-nitroacetanilide form centrosymmetric crystals whereas the other dopants used do not; ${ }^{26-30}$ no trend is seen between the two categories of crystals. While we expected piezoelectric response to increase linearly with increased dopant dipole moment, interactions between the dopant and the PDMS matrix likely increasingly hinder full alignment of larger dipoles. 

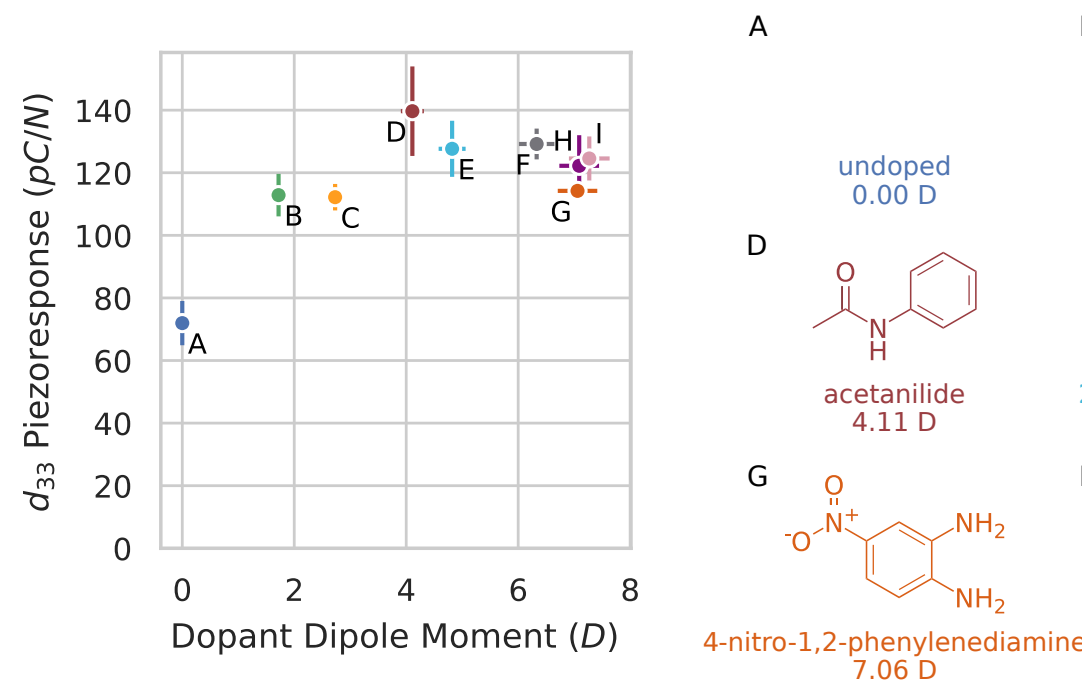

B

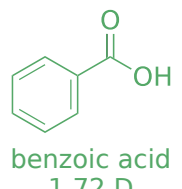

$1.72 \mathrm{D}$

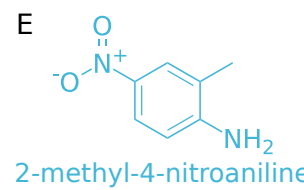

$4.82 \mathrm{D}$

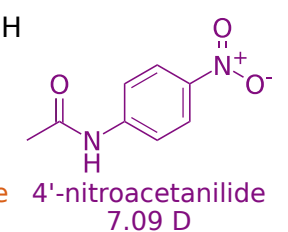

C

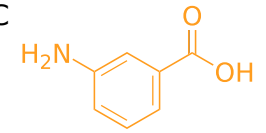

3-aminobenzoic acid $2.73 \mathrm{D}$

$\mathrm{F} \quad \mathrm{O}$

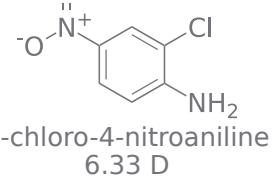

I

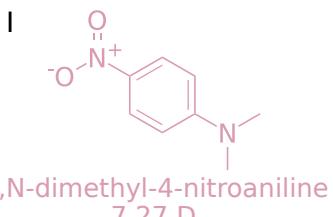

Figure 3: (A-I) Piezoelectric response as a function of dopant dipole moment for samples poled at $600 \mathrm{~V} / \mathrm{mm}$ after being doped with a $0.1 \mathrm{M}$ solution. Dipole moments are experimental values in dioxane taken from the literature. ${ }^{31-34} \mathrm{X}$-axis error bars are 5\%; Y-axis error bars represent standard error across at least four samples.

The compressive modulus of the PDMS foams is $9.24 \pm 0.14 \mathrm{kPa}$; the secant of the stress-strain curves (for strains corresponding to applied forces up to $10 \mathrm{~N}$ ) was used as no linear region was observed (Figure S4). As a result, the modulus for lesser forces is lower than the reported value. In order to evaluate the effect of modulus on piezoelectric response, the modulus of the foam needed to be varied without altering its structure. To accomplish this, the PDMS was cured at several temperatures (room temperature, $100^{\circ} \mathrm{C}$, and $200{ }^{\circ} \mathrm{C}$ ) ${ }^{35}$ a switch to a sodium chloride mold was necessary to accommodate the needed temperature range. These data are not directly comparable to the rest of this work as the foam structure is different; as these foams exhibit a linear stress-strain curve, the modulus can be determined by the slope of the linear region (Figure S5). Figure 4 displays the piezoelectric response as a function of modulus. Increasing the foam modulus lead to a decrease in the resultant piezoelectric response, as expected (see Equation 2 and our previous work ${ }^{16}$ ).

Several additional properties of the PDMS foams were studied. In Figure 5, the voltage drop and resulting generated power density are plotted as a function of resistance. The voltage and power density increase logarithmically until a peak voltage of $85 \mathrm{mV}$ and power density of $0.23 \mu \mathrm{W} / \mathrm{m}^{2}$ both corresponding to a resistance of $100 \mathrm{M} \Omega$. The stability of the piezoelectric response is poor as it decayed back to baseline after just two days (Figure S6); repoling samples boosted their 


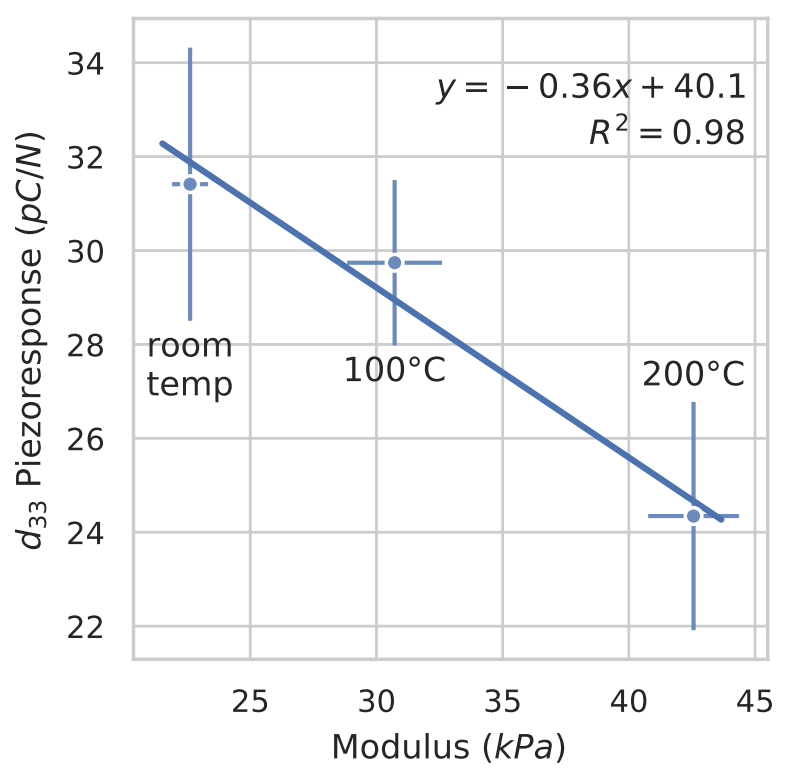

Figure 4: Piezoelectric response as a function of compressive modulus for sodium chloride templated foams doped with $0.1 \mathrm{M}$ CNA and poled at $800 \mathrm{~V} / \mathrm{mm}$. Foams were cured at room temperature, $100{ }^{\circ} \mathrm{C}$, and $200^{\circ} \mathrm{C}$ (as labeled). Error bars represent standard error; modulus was measured for a subset of four samples in each set.

piezoelectric response back to near initial levels. The piezoelectric response appears to decay in a double exponential fashion, but the decay kinetics are beyond the scope of this study. The decay in piezoelectric response is likely due to thermal randomizations as repoling restores the electrical response while mechanical properties remain constant; this decay is expected because the PDMS matrix has been fully cured prior to poling and the dopant is only dispersively adhered to the matrix surface - there is nothing to lock the dipoles in place. A key challenge for future work is to improve the stability of the piezoelectric response and to incorporate higher concentrations of dopant molecules into the polymer matrix itself - instead of just coating the foam voids.

\section{Conclusions}

We have demonstrated that the piezoelectric response of our PDMS foam PEH can be successfully tuned through changes to both its relative permittivity and its compressive modulus. By changing the poling field, the dopant (and its dipole moment), or the dopant concentration, we are able to vary 


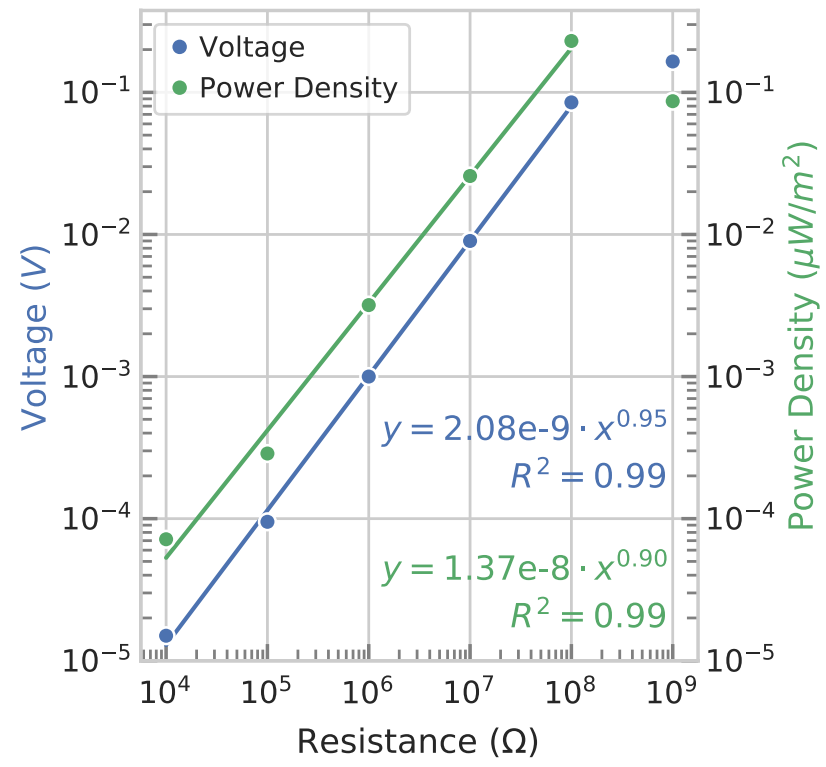

Figure 5: Voltage drop over various resistances and the resulting generated power density for a $0.1 \mathrm{M}$ CNA doped sample poled at $800 \mathrm{~V} / \mathrm{mm}$. Note the log scales.

the electrical properties of our PEH and optimize its piezoelectric response. We are similarly able to optimize the piezoelectric response by changing the mechanical properties through variations in the foam's compressive modulus. While the ideal dopant concentration varied by poling field, increasing the poling field generally produced the largest piezoelectric response. Our maximum measured piezoelectric response, a remarkable $153 \mathrm{pC} / \mathrm{N}$, was from a foam doped with $0.1 \mathrm{M}$ CNA and poled under an electric field of $800 \mathrm{~V} / \mathrm{mm}$. Mechanically, softer foams produced the highest piezoelectric response. Combined with our previous work with polyurethane foams, we have presented a highly tunable system that is general to a wide range of polymer matrices and polar dopants. Going forward, we aim to further improve the piezoelectric response of our materials and their stability by incorporating polar molecules directly into the polymer matrix through covalent attachment.

\section{Acknowledgement}

The authors thank Julia Cuthbert for assistance with taking compression measurements and Tomasz Kowalewski for use of the rheometer. The authors acknowledge the University of Pittsburgh for financial support. 


\section{Supporting Information Available}

Analysis of piezoelectric response for forces up to $10 \mathrm{~N}$; stress-strain curves for foams; stability of piezoelectric response over time $(\mathrm{PDF})$

Peak finding Python script (PY)

\section{References}

(1) Wang, Z. L. Towards Self-Powered Nanosystems: From Nanogenerators to Nanopiezotronics. Adv. Funct. Mater. 2008, 18, 3553-3567.

(2) Dagdeviren, C.; Yang, B. D.; Su, Y.; Tran, P. L.; Joe, P.; Anderson, E.; Xia, J.; Doraiswamy, V.; Dehdashti, B.; Feng, X.; Lu, B.; Poston, R.; Khalpey, Z.; Ghaffari, R.; Huang, Y.; Slepian, M. J.; Rogers, J. A. Conformal Piezoelectric Energy Harvesting and Storage from Motions of the Heart, Lung, and Diaphragm. Proc. Natl. Acad. Sci. U.S.A. 2014, 111, 1927-1932.

(3) Dagdeviren, C.; Joe, P.; Tuzman, O. L.; Park, K.-I.; Lee, K. J.; Shi, Y.; Huang, Y.; Rogers, J. A. Recent Progress in Flexible and Stretchable Piezoelectric Devices for Mechanical Energy Harvesting, Sensing and Actuation. Extreme Mech. Lett. 2016, 9, 269-281.

(4) Mader, G.; Meixner, H.; Kleinschmidt, P. Temperature and Stress Dependence of Young's Modulus in Semiconducting Barium Titanate Ceramics. J. Appl. Phys. 1985, 58, 702-704.

(5) Webber, K.; Aulbach, E.; Key, T.; Marsilius, M.; Granzow, T.; Rödel, J. TemperatureDependent Ferroelastic Switching of Soft Lead Zirconate Titanate. Acta Mater. 2009, 57, 4614-4623.

(6) Bowen, C. R.; Kim, H. A.; Weaver, P. M.; Dunn, S. Piezoelectric and Ferroelectric Materials and Structures for Energy Harvesting Applications. Energy Environ. Sci. 2014, 7, 25-44.

(7) Yan, Y.; Zhou, J. E.; Maurya, D.; Wang, Y. U.; Priya, S. Giant Piezoelectric Voltage Coefficient in Grain-Oriented Modified $\mathrm{PbTiO}_{3}$ Material. Nat. Commun. 2016, \%, 13089. 
(8) Kim, K.; Zhu, W.; Qu, X.; Aaronson, C.; McCall, W. R.; Chen, S.; Sirbuly, D. J. 3D Optical Printing of Piezoelectric Nanoparticle-Polymer Composite Materials. ACS Nano 2014, 8, 9799-9806.

(9) Anton, S.; Farinholt, K.; Erturk, A. Piezoelectret Foam-Based Vibration Energy Harvesting. J. Intell. Mater. Syst. Struct. 2014, 25, 1681-1692.

(10) Lee, J.-H.; Heo, K.; Schulz-Schönhagen, K.; Lee, J. H.; Desai, M. S.; Jin, H.-E.; Lee, S.-W. Diphenylalanine Peptide Nanotube Energy Harvesters. ACS Nano 2018, 12, 8138-8144.

(11) Zhang, Y.; Jeong, C. K.; Yang, T.; Sun, H.; Chen, L.-Q.; Zhang, S.; Chen, W.; Wang, Q. Bioinspired Elastic Piezoelectric Composites for High-Performance Mechanical Energy Harvesting. J. Mater. Chem. A 2018, 6, 14546-14552.

(12) Ma, S.; Jin, L.; Huang, X.; Riziotis, C.; Huang, R.; Zhang, C.; Lu, J.; Yang, W. Nanogenerators Begin to Light Up: A Novel Poling-Free Piezoelectric System with Multicolor Photoluminescence as an Efficient Mechatronics Development Platform. Adv. Mater. Interfaces 2018, 5, 1800587.

(13) McCall, W. R.; Kim, K.; Heath, C.; La Pierre, G.; Sirbuly, D. J. Piezoelectric Nanoparticle-Polymer Composite Foams. ACS Appl. Mater. Interfaces 2014, 6, 19504-19509.

(14) Maity, K.; Garain, S.; Henkel, K.; Schmeißer, D.; Mandal, D. Natural Sugar-Assisted, Chemically Reinforced, Highly Durable Piezoorganic Nanogenerator with Superior Power Density for SelfPowered Wearable Electronics. ACS Appl. Mater. Interfaces 2018, 10, 44018-44032.

(15) IRE Standards on Piezoelectric Crystals: Determination of the Elastic, Piezoelectric, and Dielectric Constants-The Electromechanical Coupling Factor, 1958. Proc. IRE 1958, 46, $764-778$.

(16) Moody, M. J.; Marvin, C. W.; Hutchison, G. R. Molecularly-Doped Polyurethane Foams with Massive Piezoelectric Response. J. Mater. Chem. C 2016, 4, 4387-4392.

(17) Berlincourt, D. A.; Cmolik, C.; Jaffe, H. Piezoelectric Properties of Polycrystalline Lead Titanate Zirconate Compositions. Proc. IRE 1960, 48, 220-229. 
(18) Böttcher, C. J. F.; van Belle, O. C.; Bordewijk, P.; Rip, A. Theory of Electric Polarization, 2nd ed.; Elsevier Scientific Publishing Company: New York, 1973.

(19) Newman, B. A.; Scheinbeim, J. I.; Sen, A. The Effect of Plasticizer on the Piezoelectric Properties of Unoriented Polyvinylidene Fluoride Films. Ferroelectrics 1984, 57, 229-241.

(20) Choi, S.-J.; Kwon, T.-H.; Im, H.; Moon, D.-I.; Baek, D. J.; Seol, M.-L.; Duarte, J. P.; Choi, Y.-K. A Polydimethylsiloxane (PDMS) Sponge for the Selective Absorption of Oil from Water. ACS Appl. Mater. Interfaces 2011, 3, 4552-4556.

(21) Swallowe, G. M. In Mechanical Properties and Testing of Polymers: An A-Z Reference; Swallowe, G. M., Ed.; Springer Netherlands: Dordrecht, 1999; pp 242-247.

(22) Technical Data Sheet: SYLGARD 184 Silicone Elastomer; 2017; The Dow Chemical Company; Form No. 11-3184-01 C.

(23) Tang, Y.; Zheng, Q.; Chen, B.; Ma, Z.; Gong, S. A New Class of Flexible Nanogenerators Consisting of Porous Aerogel Films Driven by Mechanoradicals. Nano Energy 2017, 38, 401-411.

(24) Chae, I.; Jeong, C. K.; Ounaies, Z.; Kim, S. H. Review on Electromechanical Coupling Properties of Biomaterials. ACS Appl. Bio Mater. 2018, 1, 936-953.

(25) Baytekin, H. T.; Baytekin, B.; Grzybowski, B. A. Mechanoradicals Created in "Polymeric Sponges" Drive Reactions in Aqueous Media. Angew. Chem. Int. Ed. Engl. 2012, 51, 3596-3600.

(26) Groom, C. R.; Bruno, I. J.; Lightfoot, M. P.; Ward, S. C. The Cambridge Structural Database. Acta Crystallogr., Sect. B: Struct. Sci., Cryst. Eng. Mater. 2016, 72, 171-179.

(27) Sim, G. A.; Robertson, J. M.; Goodwin, T. H. The Crystal and Molecular Structure of Benzoic Acid. Acta Crystallogr. 1955, 8, 157-164.

(28) Williams, P. A.; Hughes, C. E.; Lim, G. K.; Kariuki, B. M.; Harris, K. D. M. Discovery of a New System Exhibiting Abundant Polymorphism: m-Aminobenzoic Acid. Cryst. Growth Des. 2012, 12, 3104-3113. 
(29) Brown, C. J. Further Refinement of the Crystal Structure of Acetanilide. Acta Crystallogr. 1966, 21, 442-445.

(30) Coles, S. L.; Threlfall, T. L.; Hursthouse, M. B. N-(4-Nitrophenyl)acetamide. 2009; http: //ecrystals.chem.soton.ac.uk/1383/.

(31) Vaughan, W. E., Ed. Digest of Literature of Dielectrics; National Academy of Sciences: Washington, 1973; Vol. 37.

(32) Lutskii, A. E.; Kondratenko, B. P. Intramolecular Hydrogen Bond and Dipole Moments of Organic Compounds. IV. Nitro- and Acetylacetanilides. J. Gen. Chem. USSR 1959, 29, $2041-2044$.

(33) McClellan, A. L. Tables of Experimental Dipole Moments; W. H. Freeman: San Francisco, 1963.

(34) Millefiori, S.; Favini, G.; Millefiori, A.; Grasso, D. Electronic Spectra and Structure of Nitroanilines. Spectrochim. Acta, Part A 1977, 33, 21-27.

(35) Liu, M.; Sun, J.; Chen, Q. Influences of Heating Temperature on Mechanical Properties of Polydimethylsiloxane. Sens. Actuators, A 2009, 151, 42-45. 
Graphical TOC Entry

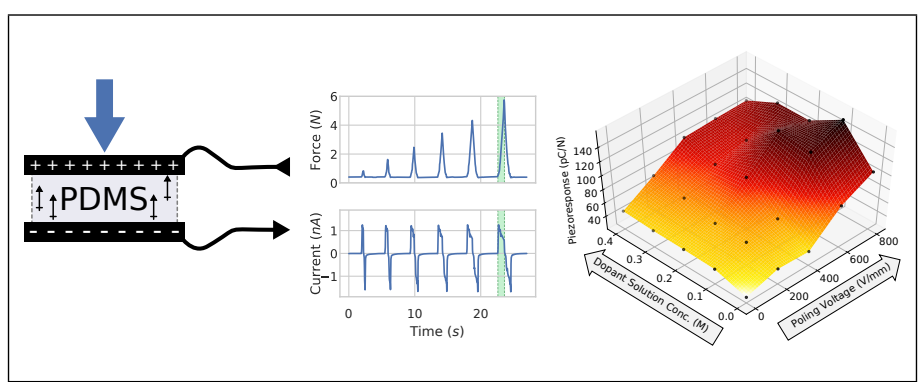




\title{
Supporting Information:
}

\section{Highly Tunable Molecularly Doped Flexible Poly(dimethylsiloxane) Foam Piezoelectric Energy Harvesters}

\author{
Christopher A. Petroff, Thomas F. Bina, and Geoffrey R. Hutchison* \\ Department of Chemistry, University of Pittsburgh, 219 Parkman Avenue, Pittsburgh, \\ Pennsylvania 15260, United States
}

E-mail: geoffh@pitt.edu 


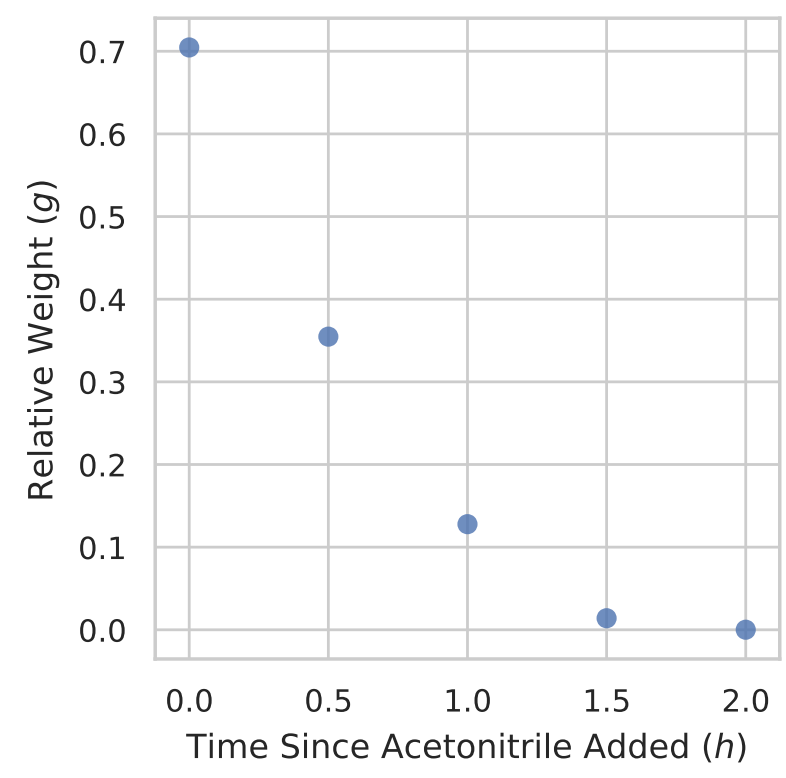

Figure S1: Evaporation rate of acetonitrile in PDMS foam. Weights are relative to the weight of the PDMS foam sample before $1 \mathrm{~mL}$ of acetonitrile was added.
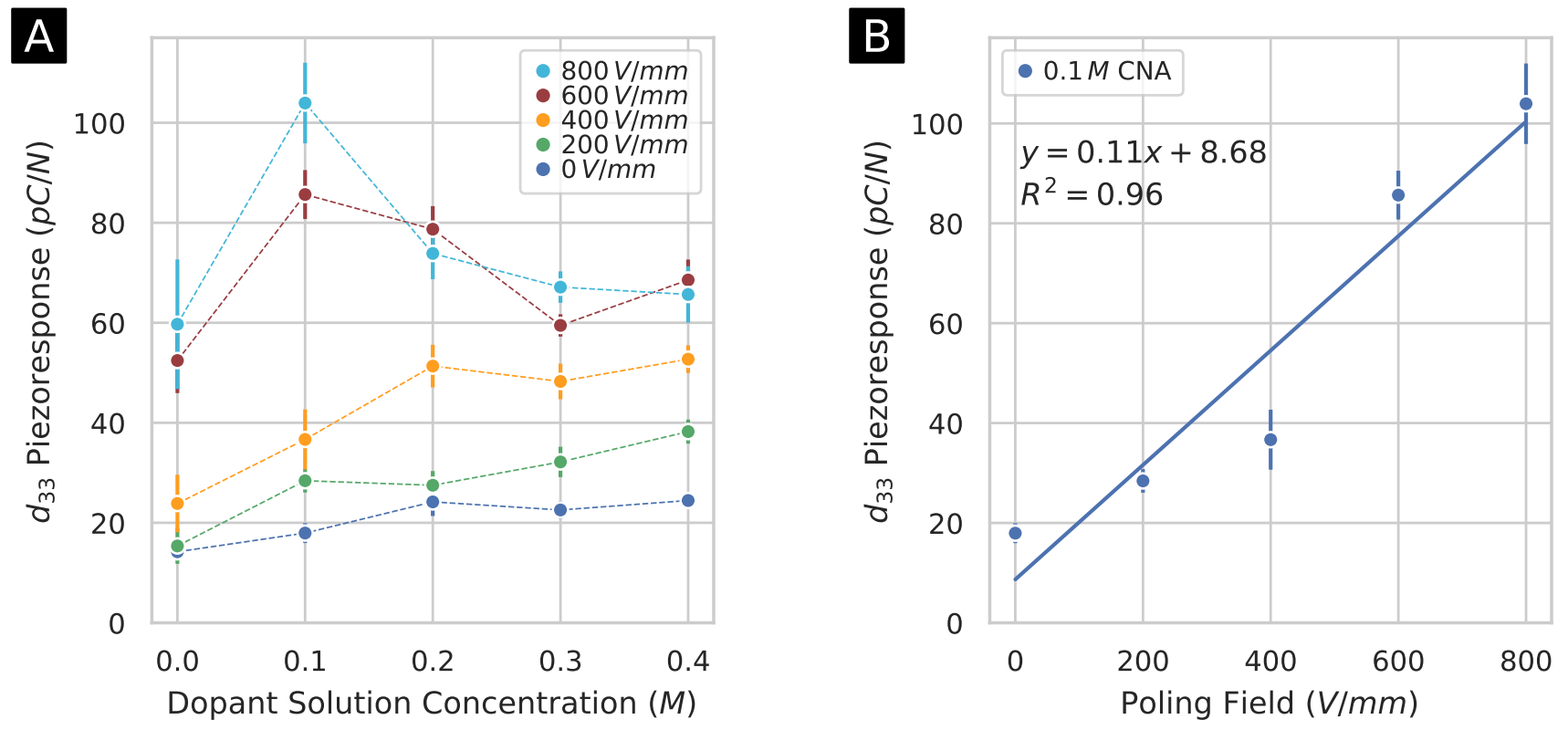

Figure S2: (A) The piezoelectric response as a function of added CNA dopant solution concentration over an array of poling fields using force values up to $10 \mathrm{~N}$. (B) The $0.1 \mathrm{M}$ CNA dopant solution subset showing the piezoelectric response increasing as a function of poling field. Error bars represent standard error across at least four samples. 


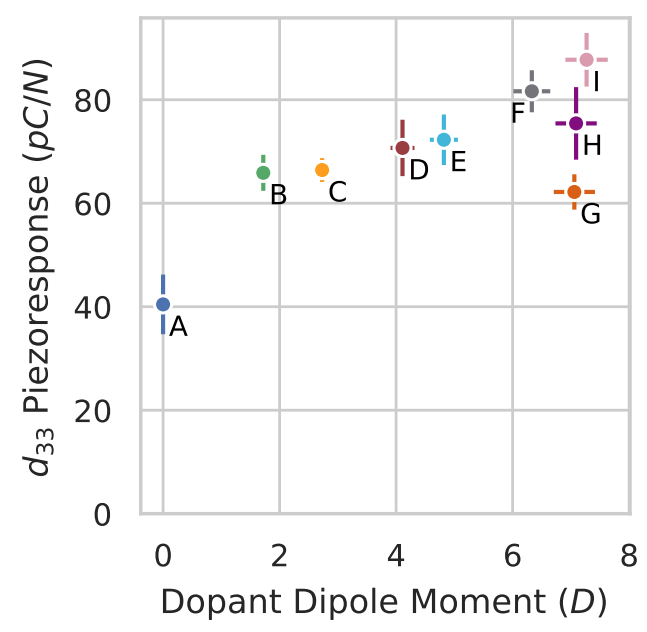

A

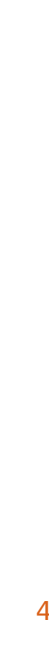

B

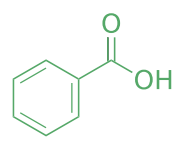

benzoic acid

$1.72 \mathrm{D}$

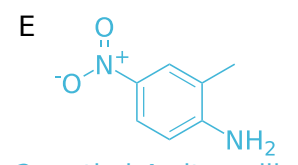

2-methyl-4-nitroaniline

4.82 D

$\mathrm{H}$
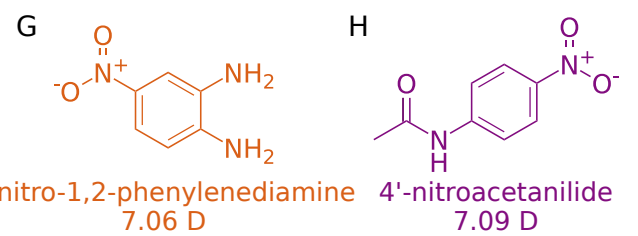
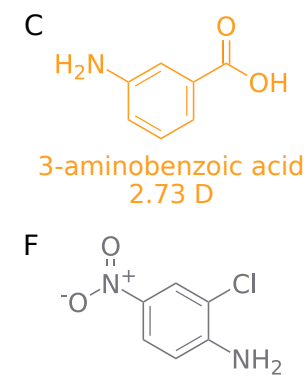

2-chloro-4-nitroaniline

$6.33 \mathrm{D}$

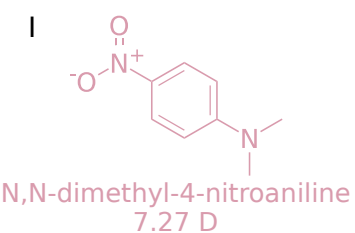

Figure S3: Piezoelectric response as a function of dopant dipole moment for samples poled at $600 \mathrm{~V} / \mathrm{mm}$ after being doped with a $0.1 \mathrm{M}$ solution using force values up to $10 \mathrm{~N}$. Dipole moments are experimental values in dioxane taken from the literature. ${ }^{\mathrm{S} 1-\mathrm{S} 4} \mathrm{X}$-axis error bars are $5 \%$; Y-axis error bars represent standard error across at least four samples.

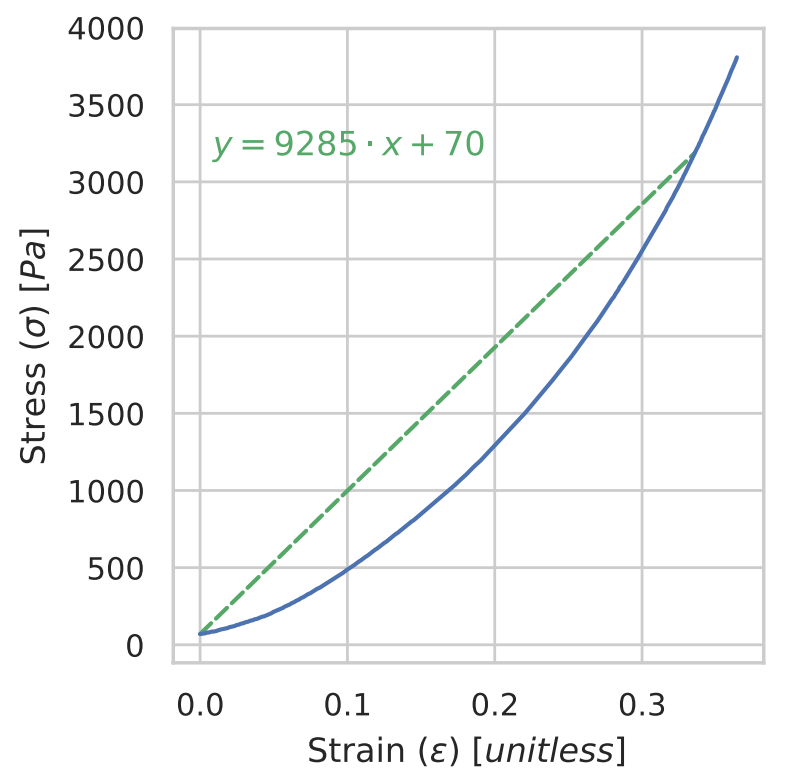

Figure S4: Stress-strain curve for sugar templated PDMS foam. Compressive modulus is the slope of the secant (dashed green line) taken from zero strain to the strain corresponding to $10 \mathrm{~N}$ of applied force. 


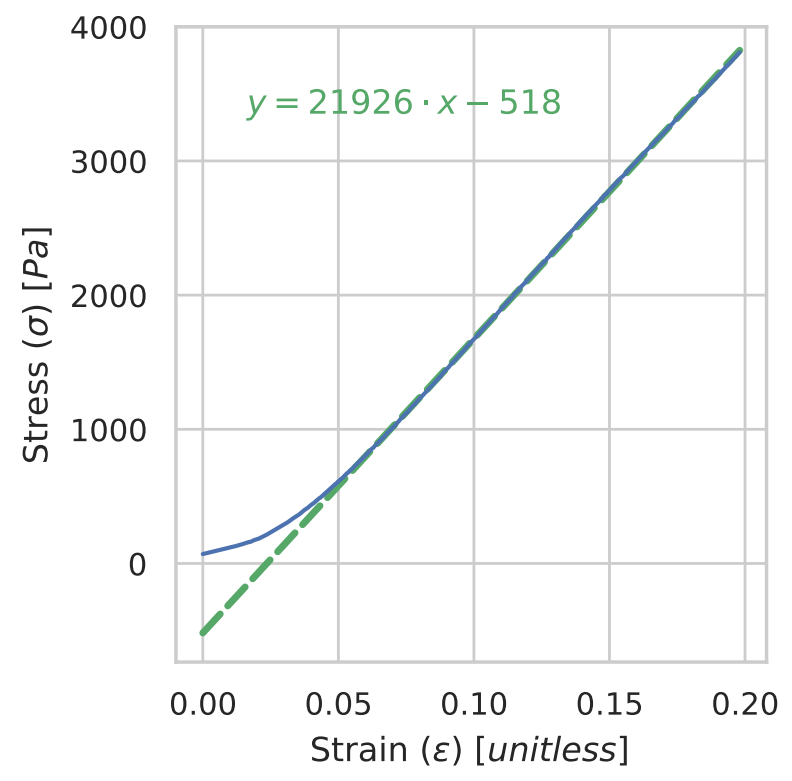

Figure S5: Stress-strain curve for a salt templated PDMS foam cured at room temperature. Compressive modulus is the slope of the linear region (dashed green line).

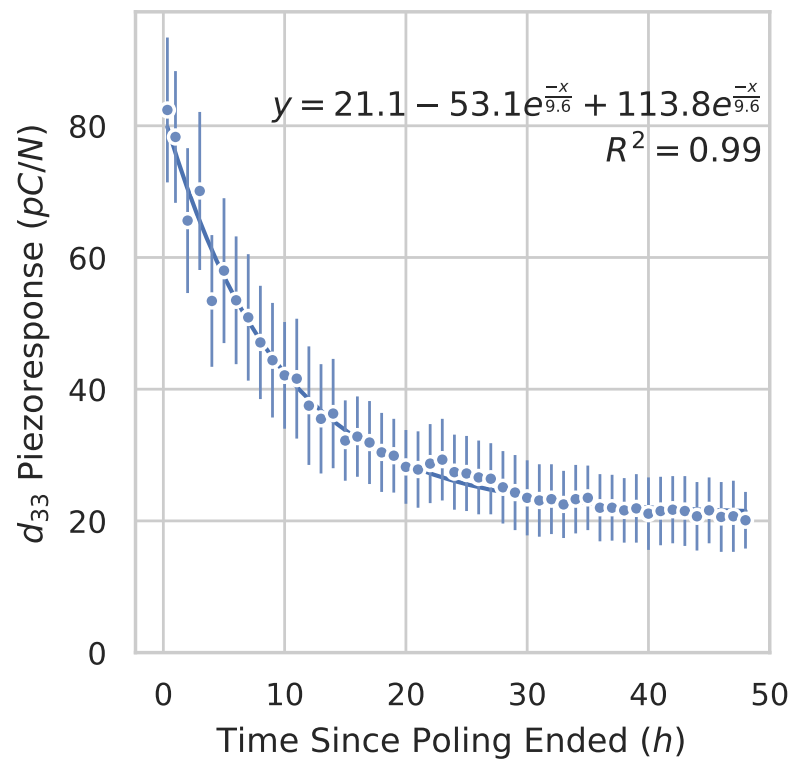

Figure S6: Decay in piezoelectric response over time for a PDMS foam sample doped with $0.4 \mathrm{M}$ CNA and poled at $800 \mathrm{~V} / \mathrm{mm}$. 


\section{References}

(S1) Vaughan, W. E., Ed. Digest of Literature of Dielectrics; National Academy of Sciences: Washington, 1973; Vol. 37.

(S2) Lutskii, A. E.; Kondratenko, B. P. Intramolecular Hydrogen Bond and Dipole Moments of Organic Compounds. IV. Nitro- and Acetylacetanilides. J. Gen. Chem. USSR 1959, 29, 2041-2044.

(S3) McClellan, A. L. Tables of Experimental Dipole Moments; W. H. Freeman: San Francisco, 1963.

(S4) Millefiori, S.; Favini, G.; Millefiori, A.; Grasso, D. Electronic Spectra and Structure of Nitroanilines. Spectrochim. Acta, Part A 1977, 33, 21-27. 
Other files

tex-files.zip (0.92 MiB)

peak-finding-script.py (9.23 KiB) view on ChemRxiv • download file

view on ChemRxiv - download file 